\title{
Carvedilol can Prevent Cardiac Events in Duchenne Muscular Dystrophy
}

\author{
Tsuyoshi Matsumura ${ }^{1}$, Takuhisa Tamura ${ }^{2}$, Satoshi Kuru ${ }^{3}$, \\ Yasuki Kikuchi ${ }^{4}$ and Mitsuru Kawai ${ }^{5}$
}

\begin{abstract}
Objective Heart failure is one of the most serious complications in Duchenne muscular dystrophy (DMD). Beta-blocker medication is known to improve the prognosis of chronic heart failure of adults, but its efficacy and safety for DMD patients has not been fully assessed. Thus we conducted a multicenter open trial. Methods Fifty-four DMD patients participated; 41 received carvedilol (BB group) and 13 did not (non BB group). All patients with an ejection fraction of less than 50\% received angiotensin-converting enzyme inhibitor. Then, patients in BB group were started on carvedilol. The mean maintenance dose of carvedilol in BB group was $7.85 \pm 2.80 \mathrm{mg} /$ day. Clinical signs and cardiac function were monitored regularly and statistical analysis was done.

Results The survival rate free from primary endpoints (death, deterioration of heart failure and severe arrhythmia) was higher in the BB group. The survival rate free from all-cause death was also higher in the BB group, although not significantly higher. Patients with primary endpoints received lower maintenance doses of carvedilol and presented higher mean heart rates (HR) during the observation period. In the BB group, mean $\mathrm{HR}$ at enrollment and the reduction of mean HR were correlated with the change of ejection fraction. Although serious adverse events were rare during the introduction of carvedilol, patients with advanced cardiac dysfunction required a longer period for up-titration and frequently presented with minor complaints.

Conclusion The present study suggests that carvedilol is relatively safe and can prevent cardiac events even in patients with DMD.
\end{abstract}

Key words: Duchenne muscular dystrophy, cardiac dysfunction, cardioprotection, angiotensin converting enzyme inhibitor, beta-blocker

(Inter Med 49: 1357-1363, 2010)

(DOI: 10.2169/internalmedicine.49.3259)

\section{Introduction}

Duchenne muscular dystrophy (DMD), one of the most common muscular dystrophies, is caused by a mutation of the dystrophin gene at Xp21. Respiratory failure and heart failure are inevitable and potentially lethal complications. Although respiratory management and infection control have improved the prognosis of DMD (1), it is not clear whether the prognosis of heart failure has been improved.
Today, approximately one-half of DMD patients in Japan die from heart failure (2). In DMD, various factors including respiratory failure, immobility, deformity obscure the clinical signs of heart failure and make difficult for accurate assessment of cardiac function. Thoracic deformity and poor acoustic windows hamper echocardiographic assessment. The sensitivity of natriuretic peptide is not always sufficient in these patients (3-6). In addition, for most DMD patients cardiologists are not sufficiently accessible for regular cardiac management. In the general population, evidence has

\footnotetext{
${ }^{1}$ Department of Neurology, National Hospital Organization Toneyama National Hospital, Toyonaka, ${ }^{2}$ Department of Internal Medicine, National Hospital Organization Higashi-Saitama National Hospital, Hasuda, ${ }^{3}$ Department of Neurology, National Hospital Organization Suzuka National Hospital, Suzuka, ${ }^{4}$ Department of Occupational Therapy, Nagasaki University School of Health Science, Nagasaki and ${ }^{5}$ Department of Neurology, National Hospital Organization Higashi-Saitama National Hospital, Hasuda
}

Received for publication December 14, 2009; Accepted for publication March 23, 2010

Correspondence to Dr. Tsuyoshi Matsumura, matumura@toneyama.hosp.go.jp 
accumulated regarding the benefits of angiotensin-converting enzyme inhibitors (ACEIs) $(7,8)$, angiotensin II type 1 receptor blockers (ARBs) (9-12), and beta-blockers (13-19) for chronic heart failure. Recently, ACEIs have been recognized as the primary agents for cardiac dysfunction in patients with muscular dystrophies $(20,21)$. Although betablockers have been gradually used in heart failure for these patients, they have not become as popular as ACEIs/ARBs. The concern about adverse effects $(22,23)$ of beta-blockers and the lack of sufficient evidence for their use in children $(24,25)$ and in muscular dystrophies $(21,26)$ deters physicians from prescribing them. Considering the nature of DMD, cardiac function should be monitored regularly from diagnosis of the disease and cardioprotective therapy should be initiated from an early stage of cardiac dysfunction $(5,21,26)$. In addition, it is also important for the general physician to design a safe way to titrate beta-blockers in advanced cases (23). We therefore conducted a prospective multicenter trial to assess the effectiveness and safety of carvedilol in DMD.

\section{Subjects}

The protocol of this study was reviewed and approved by the ethics committee in each center. Subjects were DMD patients who were managed at our hospitals and provided informed consent (obtained from parents or guardians for juvenile patients). The following exclusion criteria were applied: acute heart failure, active asthma, severe uncontrolled arrhythmia, advanced atrioventricular block, therapy with agents influencing the sympathetic nervous system, bradycardia $<50$ beats per minutes (bpm), systolic blood pressure $<80 \mathrm{mmHg}$, severe structural deformity precluding ultrasound cardiography (UCG), and contraindications to ACEIs, ARBs, or beta-blockers. Fifty-four patients were eventually enrolled in this study.

For ethical reasons, allocation to groups was performed in an open manner and 49 patients chose their own treatments. Forty-one patients were assigned to treatment with carvedilol (BB group) and 13 were assigned to treatment without carvedilol (non BB group). No participants had complaints associated with cardiac dysfunction at enrollment.

\section{Methods}

\section{Study design}

When LVEF was less than 50\%, all patients received ACEIs or ARBs. Carvedilol was introduced in the BB group after patients were stable on ACEIs or ARBs for at least one month. Since many patients had low body weight (less than $35 \mathrm{~kg}$ ) and our previous study of patients with advanced cardiac dysfunction (LVEF <40\%) showed frequent minor adverse effects during titration of a beta-blocker (23), carvedilol was started at a low dose $(0.3125 \mathrm{mg}$ or $0.625 \mathrm{mg}$ twice daily) and gradually increased under careful monitor- ing of vital signs and symptoms. Most patients were hospitalized for commencement of carvedilol.

Because symptoms of heart failure are often obscure and the sensitivity of standard indexes of cardiac function is not sufficient in DMD patients, we sometimes experienced acute exacerbation of heart failure caused by minor stress when their LVEF below $30 \%$. These facts made us uneasy to introduce a beta-blocker. One of the purposes of this study was to establish a safe way to introduce a beta-blocker. In general, pimobendan and/or diuretics are used for advanced patients who cannot tolerate the introduction of betablocker $(27,28)$. Long-term effect and safety have been reported with pimobendan (29). Thus, preceding treatment with pimobendan (1.25-5.0 $\mathrm{mg} /$ day) and/or diuretics were permitted in patients with LVEF below $30 \%$ to lessen the risk of initiation of carvedilol (27-29). Because the typical adult dose of carvedilol for chronic heart failure is $5-20 \mathrm{mg} /$ day in Japan, the maintenance dose was targeted at $10 \mathrm{mg}$ / day, but doses from 5-20 mg/day were allowed. The observation period started at the initiation of carvedilol and the introduction period was assessed as the duration required for up-titration.

For patients in the non $\mathrm{BB}$ group, the observation period started at the time of enrollment or at the initiation of ACEIs/ARBs.

Assessments were performed at enrollment, in the $3 \mathrm{rd}$ month and every 6 months during the observation period, up to 5 years. Patients were asked about adverse effects and subjective symptoms, and general physical examination was performed each time. Brain natriuretic peptide (BNP), mean HR (from Holter ECG) and left ventricular ejection fraction (LVEF) measured by UCG (30) were also checked simultaneously.

\section{Study endpoints}

Patients were considered to have dropped out if they fulfilled any of the following criteria: inability to reach the maintenance dose of carvedilol (5-20 mg/day) within 3 months, suspension of carvedilol and/or ACEIs/ARBs for more than 15 days, request to change treatments, or inability to adhere to treatment because of severe adverse effects. The primary endpoints were all-cause death, deterioration heart failure requiring intravenous catecholamine, phosphodiesterase III inhibitor or diuretics more than 15 days and severe arrhythmia requiring cardioversion or intravenous antiarrhythmic agents more than 15 days. Even after any events, assessments were continued as long as possible. The secondary endpoints were changes in LVEF and BNP.

All data were sent to a registration office, where statistical analyses were performed. A safety monitoring board supervised the study and monitored severe adverse events.

\section{Statistical analysis}

Student's t test was used to compare data between the two groups. A paired t test was used for longitudinal comparisons of LVEF, BNP level, and mean HR. Fisher's exact 
Table 1. Clinicodemographic Profiles of Participants at Enrollment

\begin{tabular}{|c|c|c|c|c|c|}
\hline & \multicolumn{2}{|r|}{ non BB } & \multicolumn{2}{|r|}{$\mathrm{BB}$} & \multirow[b]{2}{*}{$\mathrm{p}$} \\
\hline & $\mathrm{n}$ & mean $\pm \mathrm{SD}$ (range) & $\mathrm{n}$ & mean $\pm \mathrm{SD}$ (range) & \\
\hline Age (y.o.) & 13 & $23.2 \pm 8.5(15-35)$ & 41 & $19.3 \pm 4.7(11-29)$ & 0.041 \\
\hline LVEF (\%) & 13 & $40.4 \pm 14.9(10-58)$ & 41 & $38.1 \pm 14.7(9-67)$ & 0.624 \\
\hline $\log (\mathrm{BNP})(\mathrm{pg} / \mathrm{mL})$ & 12 & $1.02 \pm 0.54(0.48-2.41)$ & 40 & $1.25 \pm 0.60(0.41-2.48)$ & 0.215 \\
\hline Mean HR (bpm) & 9 & $94.8 \pm 11.9(78-116)$ & 38 & $91.7 \pm 11.3(70-117)$ & 0.490 \\
\hline
\end{tabular}

Table 2. Profile of Events

\begin{tabular}{lll}
\hline & non BB & BB \\
\hline Primary events & 7 & 4 \\
All-cause death & 3 & 3 \\
Deterioration of cardiac failure & $5(2)$ & $4(3)$ \\
Arrhythmic events & 1 & 0 \\
\hline Drop out & 2 & 3 \\
\hline
\end{tabular}

The number in the parentheses refers to the number of mortality.

test was used to compare the frequency of primary endpoints. Because most participants chose their treatments, selection bias was inherent in the design. To overcome this problem, Cox regression analyses for primary endpoints and all-cause death were performed using treatment, age at enrollment, LVEF at enrollment, and logarithm of BNP at enrollment as covariates. Analysis of covariance (ANCOVA) with age at enrollment, LVEF at enrollment, and logarithm of BNP at enrollment as covariates was also used for comparison of LVEF, BNP, and mean HR during the observation period. Pearson's correlation index was used to assess correlations. Data are presented as mean \pm SD unless otherwise indicated.

\section{Results}

The clinicodemographic profiles of participants at enrollment are listed in Table 1. Mean age of the non BB group was higher than that of the BB group. However, there were no significant differences in LVEF, logarithm of $\mathrm{BNP}$, or mean HR between the groups.

The mean observation period was $1,444 \pm 608$ days in non BB group and $1,385 \pm 470$ days in BB group $(p=0.751)$. Eleven patients experienced primary endpoints (non BB group: 7 (53.8\%), mean age, 22.0 7.3 ; BB group: 4 (9.8\%), mean age, $20.3 \pm 4.3$ ) and six patients died (non BB group: 3 (23.1\%), mean age, $18.7 \pm 5.5$; BB: 3 (7.3\%), mean age, $19.0 \pm 4.4)$ during the observation period. The mean age of patients having primary endpoints was not different $(\mathrm{p}=$ 0.631 ) between the two groups. Fisher exact test showed a significant difference in the frequency of primary endpoints $(\mathrm{p}=0.002)$, although not in the mortality $(\mathrm{p}=0.143)$. Deterioration of heart failure was observed in 9 patients (non BB group: 5, mean age, 23.6 \pm 8.0 ; BB group: 4, mean age, $20.2 \pm 4.3$ ) and 5 patients died from heart failure. Atrial flutter requiring catheter ablation was observed in a non BB group patient. Two non BB group patients developed critical incidents requiring cardiopulmonary resuscitation at their home from uncertain causes. One patient died and the other patient survived in a vegetable state. The surviving patient was not included in the primary endpoint because we could not specify the actual cause (Table 2).

Five patients dropped out of this study (Table 2). Two non BB group patients wished to take carvedilol in the fourth and the fifth year after enrollment, respectively. One BB group patient with advanced cardiac dysfunction (LVEF $11.2 \%$ ) stopped carvedilol immediately because of worsening general fatigue after starting this agent. Another BB group patient suspended carvedilol for 20 days because he was prohibited oral intake due to repeated aspiration pneumonia. The remaining patient (in the BB group) could not reach the maintenance dose within three months because of bradycardia.

According to Cox regression analyses, the survival rate free from primary endpoints was significantly higher in the BB group (Fig. 1A). Survival rate free from all-cause death was also higher in the BB group, although this difference was not significant (Fig. 1B). Compared with patients free from primary endpoints, those with primary endpoints had a lower maintenance dose of carvedilol $(3.18 \pm 6.73 \mathrm{mg} /$ day versus $6.73 \pm 3.78 \mathrm{mg} /$ day, $\mathrm{p}=0.034)$ and higher mean $\mathrm{HR}$ during the observation period $(89.8 \pm 17.2 \mathrm{bpm}$ versus $80.9 \pm$ $8.6 \mathrm{bpm}, \mathrm{p}=0.036$ ).

During the study period, there were no differences in LVEF or logarithm of BNP between the two groups. Furthermore, no significant longitudinal changes were detected in either group, with the exception of BNP, which was higher in the 54th month in the non $\mathrm{BB}$ group (Fig. 2A, 2B). As mentioned above, there was no difference in mean HR between the non BB and BB groups at enrollment. However, carvedilol decreased mean HR, which remained low during the observation period in the BB group. Thus, significant differences were detected in mean HR between the two groups at the 6th, 18th, 24th, 30th, and 36th months (Fig. 2C). The reduction of mean HR after introduction of carvedilol was correlated with the dose of carvedilol $(\mathrm{r}=-0.474, \mathrm{p}=0.002)$.

We further analyzed the relation between changes of LVEF and HR in BB group. When we examined the differences between data at enrollment and average from the 3rd to 12th months, mean HR at enrollment was correlated with LVEF change (Fig. 3A). In addition, the reduction of mean HR was also correlated with LVEF change (Fig. 3B). The magnitude of BNP change, however, was too small for a 

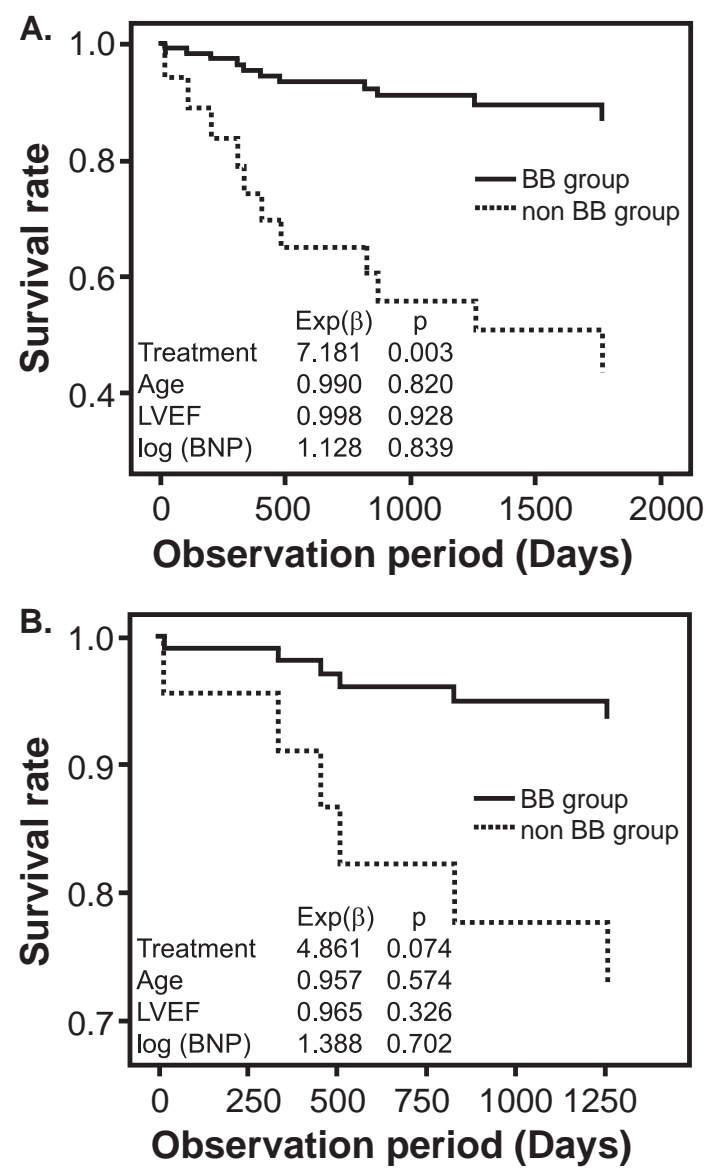

Figure 1. Cox regression analyses for cardiac events.

A. Survival rate free from cardiac events. B. Survival rate free from all-cause death. Cox regression analyses were performed using treatment, age at enrollment, left ventricular ejection fraction (LVEF) at enrollment and logarithm of brain natriuretic peptide (BNP) level at enrollment as covariates. BB: beta-blocker group, non BB: non betablocker group

similar analysis.

No patient presented with severe adverse events requiring intensive therapy during the introduction period. However minor complaints such as mild fatigue, faintness, headache, increase in expectorated sputum, and respiratory infection were frequent, especially in patients with advanced cardiac dysfunction. However, it is likely that not all of these adverse effects were caused by carvedilol.

The length of the introduction period was correlated with measures of cardiac function such as LVEF and BNP level (Fig. 4). Ten patients abandoned up-titration (for reasons including fear of adverse effects and limited hospital stay). Accordingly, the maintenance dose was less than $10 \mathrm{mg} /$ day for 15 patients $(5 \mathrm{mg} /$ day: $\mathrm{n}=13,7.5 \mathrm{mg} /$ day: $\mathrm{n}=2)$. Nine patients (LVEF 9-26\%) received pimobendan. All of these patients could reach the maintenance dose of carvedilol safely, although three of them presented minor complaints.

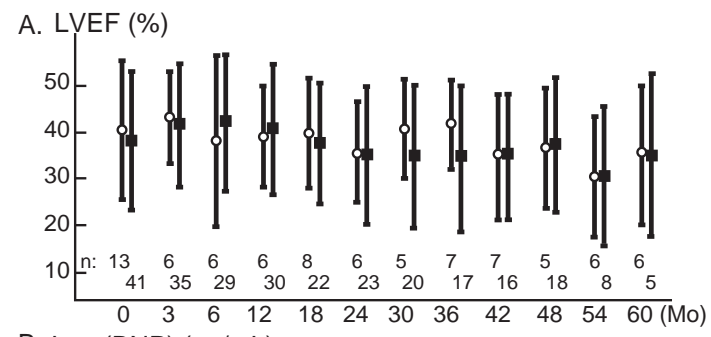

B. $\log (B N P)(p g / m L)$

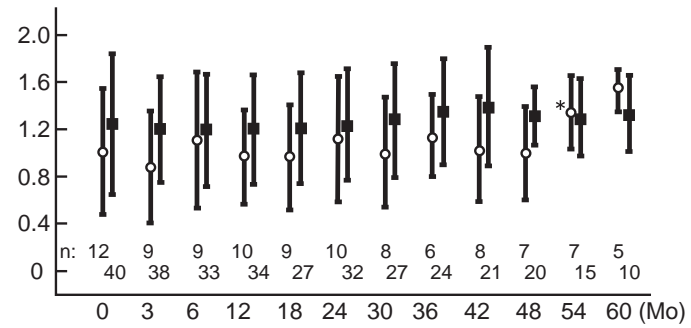

C. mean $\mathrm{HR}$ (bpm)

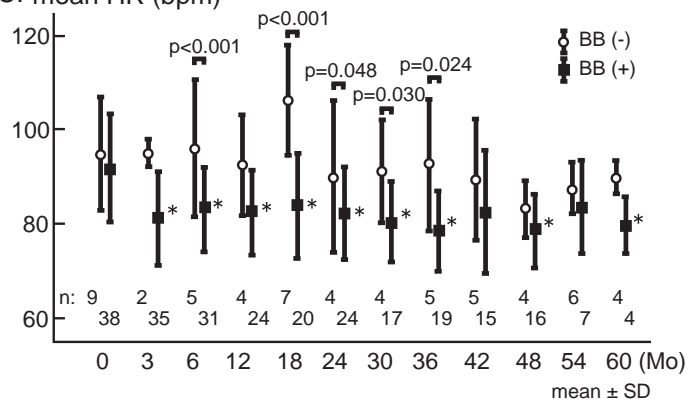

Figure 2. Longitudinal comparison of cardiac indices.

Inter-treatment comparison of LVEF (A), logarithm of BNP level (B) and mean heart rate (HR) (C) for each period was performed using analysis of covariance (ANCOVA), with age at enrollment, LVEF at enrollment and logarithm of BNP level at enrollment as covariates. $P$ values above the error bars indicate significant differences. In both treatment groups, data during the period of interest were compared to those at enrollment with a paired $t$ test. A significant difference is indicated by an asterisk beside the center point. BB: beta-blocker group, non BB: non beta-blocker group, Mo: months

\section{Discussion}

Many large studies have proven the cardioprotective effects of beta-blockers for adults with chronic heart failure (13-19). However, data regarding beta-blocker use in children $(24,25)$ or in patients with muscular dystrophies $(21,26)$ are not sufficient to establish a consensus. In this study, we prospectively assessed the effectiveness and safety of carvedilol in 54 DMD patients. For ethical reasons, patients were permitted to select their treatment, hence bias was inherent in the design. Nevertheless it is important that Cox regression analysis after correction for age, LVEF, and BNP level showed that the BB group had a higher survival rate free from cardiac events when compared with the non BB group.

In agreement with a previous study (31), the effect of 

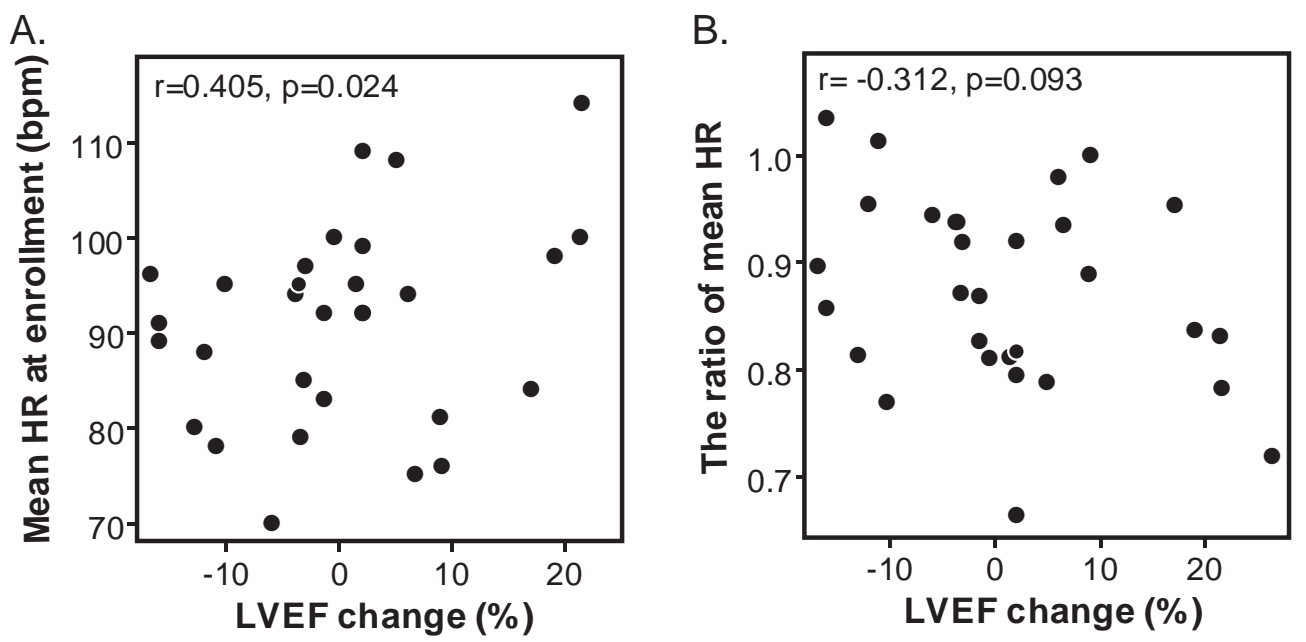

Figure 3. Correlation between heart rate and left ventricular ejection fraction change. A. Correlation between mean HR at enrollment and LVEF change. B. Correlation between the ratio of mean HR from the 3rd to the 12th month to mean HR at enrollment and LVEF change. LVEF change was calculated by subtracting LVEF at enrollment from average LVEF across the 3rd to 12th month. HR ratio shown in the $y$-axis was assessed by division of average HR from the 3rd to 12th month by mean HR at enrollment. The correlation was analyzed with Pearson's correlation index.

A.

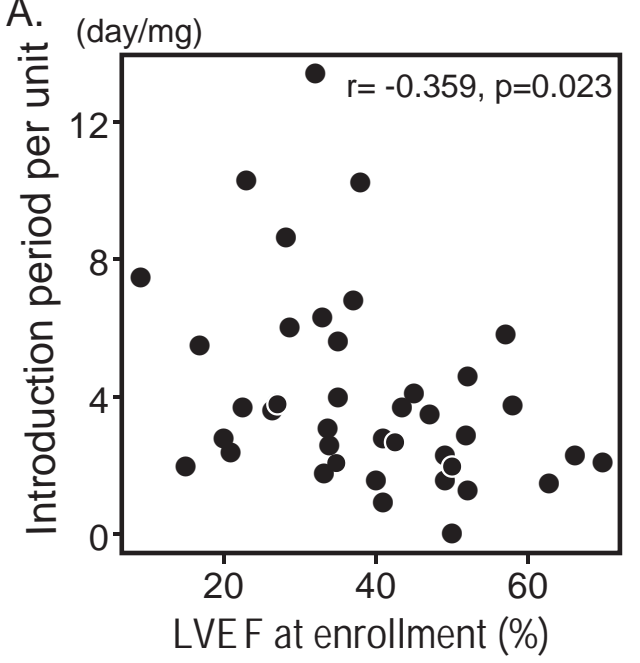

B.

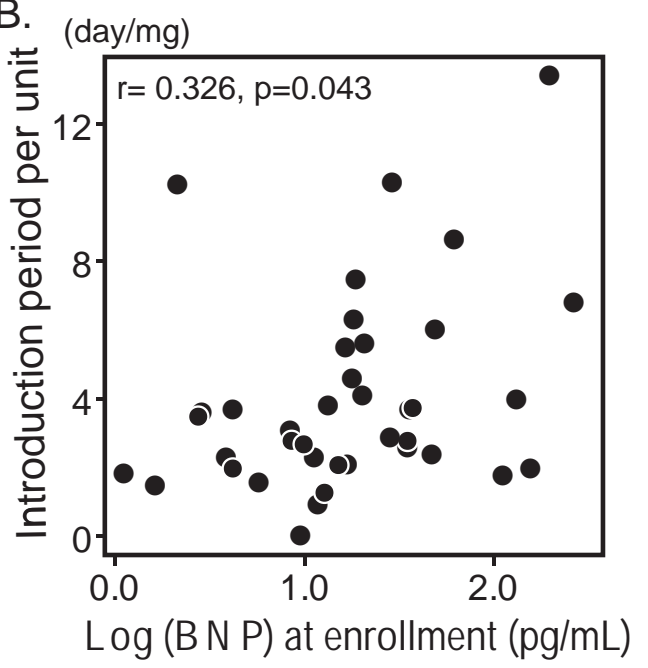

Figure 4. Correlation between the introduction period per unit dose and cardiac dysfunction. A. Correlation between introduction period per unit dose and LVEF at enrollment. B. Correlation between introduction period per unit dose and logarithm of BNP at enrollment. Introduction period per unit dose was calculated by dividing the introduction period by the maintenance dose.

carvedilol was associated with HR reduction. In the present study, patients free from primary endpoints had greater doses of carvedilol and a lower mean HR. In addition, LVEF improvement was observed in patients who showed tachycardia at enrollment and had great HR reduction with carvedilol. It seemed that a beta-blocker is more effective in patients with hyperactivity of the sympathetic nervous system. In this meaning, HR appears to be important in the indication and titration of beta-blockers since it reflects sympathetic nervous system activity.

This study could not detect obvious changes in LVEF or BNP level. Because the main purpose of this study was pre- vention of heart failure, most participants of this study were in early stage of cardiac dysfunction at enrollment. Thus, their mean BNP was within the normal range and LVEF was relatively preserved. We speculated that it was very difficult to gain obvious functional improvements in such patients. One reason may be derived from the lower sensitivities of BNP and LVEF in patients with DMD, due to immobility, muscle wasting, ventilatory management, deformity, poor acoustic windows and so on $(3,4)$. Another reason may be that the dose of carvedilol used in this study was insufficient to obtain significant functional changes, because other studies of DMD patients using higher doses of carve- 
dilol reported functional improvements $(21,26,32)$. A large study of Japanese patients with chronic heart failure (the MUCHA study) also reported that low-dose carvedilol can improve the prognosis; however, functional changes were dose-dependent (19). These findings suggest that we should maximize the dose of carvedilol to obtain functional improvement when patients can tolerate it. Even when patients cannot take enough carvedilol for functional improvement, we should continue administration because it may still improve the prognosis.

The severity of cardiac dysfunction was associated with the length of the introduction period because minor complaints were frequent, especially in patients with advanced cardiac dysfunction. Some patients abandoned up-titration because they were afraid of adverse effects or wished to shorten their hospital stay. Nonetheless, severe adverse events requiring intensive therapy during the introduction period were not reported in this study and only one patient withdrew. This suggests that the risk of beta-blockers is not as serious as previously speculated (31) although careful monitoring and gradual up-titration is essential, particularly in patients with advanced cardiac dysfunction. Coadministration of pimobendan may be useful to reduce the risk during the introduction of beta blocker in these patients.

In DMD, cardiac dysfunction invariably arises in or before the second decade of life. Most of these patients are students, and hospitalization causes profound interference with their school lives. Considering the relatively small risk in starting beta-blockers $(17,22,31)$, we recommend that these agents are begun on an outpatient basis from the early stages of cardiac dysfunction. Further studies are essential to optimize the protocol for beta-blocker treatment in patients with DMD.

\section{Acknowledgement}

We are grateful to Dr. Yoshio Yasumura, Shigeyuki Echigo and Toshio Nakanishi for their suggestions and encouragement. This study was supported by Research Grants for Nervous and Mental Disorders (14A-5, 17A-8) and Cardiovascular Disease (13C-2) from the Ministry of Health, Labour and Welfare of Japan.

\section{References}

1. Gomez-Merino E, Bach JR. Duchenne muscular dystrophy: prolongation of life by noninvasive ventilation and mechanically assisted coughing. Am J Phys Med Rehabil 81: 411-415, 2002.

2. Tatara K, Shinno S. Management of mechanical ventilation and prognosis in Duchenne muscular dystrophy. Iryo 62: 566-571, 2008.

3. Mori K, Manabe T, Nii M, Hayabuchi Y, Kuroda Y, Tatara K. Plasma levels of natriuretic peptide and echocardiographic parameters in patients with Duchenne's progressive muscular dystrophy. Pediatr Cardiol 23: 160-166, 2002.

4. Demachi J, Kagaya Y, Watanabe J, et al. Characteristics of the increase in plasma brain natriuretic peptide level in left ventricular systolic dysfunction, associated with muscular dystrophy in comparison with idiopathic dilated cardiomyopathy. Neuromuscul Disord 14: 732-739, 2004

5. Matsumura T, Saito T, Fujimura H, Shinno S. Cardiac troponin I for accurate evaluation of cardiac status in myopathic patients. Brain Dev 29: 496-501, 2007.

6. van Bockel EA, Lind JS, Zijlstra JG, et al. Cardiac assessment of patients with late stage Duchenne muscular dystrophy. Neth Heart J 17: 232-237, 2009.

7. The SOLVD Investigators. Effect of enalapril on survival in patients with reduced left ventricular ejection fractions and congestive heart failure. N Engl J Med 325: 293-302, 1991.

8. Kjekshus J, Swedberg K, Snapinn S. Effects of enalapril on longterm mortality in severe congestive heart failure. CONSENSUS Trial Group. Am J Cardiol 69: 103-107, 1992.

9. Pitt B, Segal R, Martinez FA, et al. Randomised trial of losartan versus captopril in patients over 65 with heart failure (Evaluation of Losartan in the Elderly Study, ELITE). Lancet 349: 747-752, 1997.

10. Pitt B, Poole-Wilson PA, Segal R, et al. Effect of losartan compared with captopril on mortality in patients with symptomatic heart failure: randomised trial--the Losartan Heart Failure Survival Study ELITE II. Lancet 355: 1582-1587, 2000.

11. Cohn JN, Tognoni G. A randomized trial of the angiotensinreceptor blocker valsartan in chronic heart failure. N Engl J Med 345: 1667-1675, 2001.

12. Pfeffer MA, Swedberg K, Granger CB, et al. Effects of candesar- tan on mortality and morbidity in patients with chronic heart failure: the CHARM-Overall programme. Lancet 362: 759-766, 2003.

13. Waagstein F, Bristow MR, Swedberg K, et al. Beneficial effects of metoprolol in idiopathic dilated cardiomyopathy. Metoprolol in Dilated Cardiomyopathy (MDC) Trial Study Group. Lancet 342: 1441-1446, 1993.

14. Packer M, Bristow MR, Cohn JN, et al. The effect of carvedilol on morbidity and mortality in patients with chronic heart failure. U.S. Carvedilol Heart Failure Study Group. N Engl J Med 334: 1349-1355, 1996.

15. Effect of metoprolol CR/XL in chronic heart failure: Metoprolol CR/XL Randomised Intervention Trial in Congestive Heart Failure (MERIT-HF). Lancet 353: 2001-2007, 1999.

16. The Cardiac Insufficiency Bisoprolol Study II (CIBIS-II). A randomised trial. Lancet 353: 9-13, 1999.

17. Packer M, Coats AJ, Fowler MB, et al. Effect of carvedilol on survival in severe chronic heart failure. N Engl J Med 344: 16511658, 2001.

18. Remme WJ, Riegger G, Hildebrandt $P$, et al. The benefits of early combination treatment of carvedilol and an ACE-inhibitor in mild heart failure and left ventricular systolic dysfunction. The carvedilol and ACE-inhibitor remodeling mild heart failure evaluation trial (CARMEN). Cardiovasc Drugs Ther 18: 57-66, 2004.

19. Hori M, Sasayama S, Kitabatake A, et al. Low-dose carvedilol improves left ventricular function and reduces cardiovascular hospitalization in Japanese patients with chronic heart failure: the Multicenter Carvedilol Heart Failure Dose Assessment (MUCHA) trial. Am Heart J 147: 324-330, 2004.

20. Tamura $T$, Shibuya $T$, Iida M, Ishihara $T$, Fukunaga $H$, Kyo $S$. Clinical evaluation of captopril in chronic cardiac dysfunction in patients with Duchenne muscular dystrophy. Rinsho Iyaku 12: 3635-3646, 1996.

21. Ishikawa Y, Bach JR, Minami R. Cardioprotection for Duchenne's muscular dystrophy. Am Heart J 137: 895-902, 1999.

22. Abraham WT. Beta-blockers: the new standard of therapy for mild heart failure. Arch Intern Med 160: 1237-1247, 2000.

23. Saito T, Matsumura T, Miyai I, Nozaki S, Shinno S. Carvedilol effectiveness for left ventricular-insufficient patients with Duchenne muscular dystrophy. Rinsho Shinkeigaku 41: 691-694, 2001. 
24. Bruns LA, Chrisant MK, Lamour JM, et al. Carvedilol as therapy in pediatric heart failure: an initial multicenter experience. J Pediatr 138: 505-511, 2001.

25. Shaddy RE, Boucek MM, Hsu DT, et al. Carvedilol for children and adolescents with heart failure: a randomized controlled trial. JAMA 298: 1171-1179, 2007.

26. Rhodes J, Margossian R, Darras BT, et al. Safety and efficacy of carvedilol therapy for patients with dilated cardiomyopathy secondary to muscular dystrophy. Pediatr Cardiol 29: 343-351, 2008.

27. Yoshikawa T, Baba A, Suzuki M, et al. Effectiveness of carvedilol alone versus carvedilol + pimobendan for severe congestive heart failure. For the Keio Interhospital Cardiology Study (KICS) Group. Am J Cardiol 85: 1495-1497; A1497, 2000.

28. Takeda N, Ohtaki E, Misu K, et al. Normalization of left ventricular parameters following combined pimobendan and carvedilol treatment in a case of unclassified cardiomyopathy with longstanding refractory status. Intern Med 41: 1147-1152, 2002.

29. Effects of pimobendan on adverse cardiac events and physical activities in patients with mild to moderate chronic heart failure: the effects of pimobendan on chronic heart failure study $(\mathrm{EPOCH}$ study). Circ J 66: 149-157, 2002.

30. Schiller NB, Shah PM, Crawford M, et al. Recommendations for quantitation of the left ventricle by two-dimensional echocardiography. American Society of Echocardiography Committee on Standards, Subcommittee on Quantitation of Two-Dimensional Echocardiograms. J Am Soc Echocardiogr 2: 358-367, 1989.

31. Nohria A, Lewis E, Stevenson LW. Medical management of advanced heart failure. Jama 287: 628-640, 2002.

32. Kajimoto $H$, Ishigaki $K$, Okumura $K$, et al. Beta-blocker therapy for cardiac dysfunction in patients with muscular dystrophy. Circ $\mathbf{J}$ 70: 991-994, 2006.

(C) 2010 The Japanese Society of Internal Medicine http://www.naika.or.jp/imindex.html 